\title{
Negative Poisson ratio in two-dimensional networks under tension
}

\author{
David H. Boal \\ Department of Physics, Simon Fraser University, Burnaby, British Columbia, Canada V5A 1 S6 \\ Udo Seifert \\ Institut für Festkörperforschung, Forschungszentrum Jülich, 52425 Jülich, Germany \\ Julian C. Shillcock \\ Department of Physics, Simon Fraser University, Burnaby, British Columbia, Canada V5A 1S6
}

(Received 15 June 1993)

\begin{abstract}
The elastic properties of two-dimensional networks under tension are studied by the mean-field approximation and Monte Carlo simulation. The networks are characterized by fixed (polymerized) connectivity and either a square-well or a Hooke's-law interaction among their components. Both selfavoiding and phantom networks are examined. The elastic properties of Hooke's-law networks at large equilibrium length are found to be well represented by a mean-field model. All the networks investigated show a negative Poisson ratio over a range of tension. At finite tension, the phantom networks exhibit a phase transition to a collapsed state.
\end{abstract}

PACS number(s): 64.60. $-\mathrm{i}, 05.40 .+\mathrm{j}, 62.20 . \mathrm{Dc}$, 87.22.Bt

\section{INTRODUCTION}

Two-dimensional sheets or membranes have been used to model the physical properties of a variety of systems ranging from cells in biophysics [1] to particles in highenergy physics [2]. The fundamental material parameters of these membranes are their elastic constants, such as the compression and shear modulus, as well as the bending rigidity. These quantities have been investigated theoretically both for homogeneous [3-7] as well as heterogeneous [8-12] membranes.

A particularly interesting quantity of any elastic body is its Poisson ratio $\sigma$, which is constructed from the bulk and shear moduli. The Poisson ratio is a measure of the strain in a transverse direction which results from a stress applied longitudinally. In almost all known materials, the experimentally measured Poisson ratio is positive, which corresponds to a material which shrinks transversely when stretched longitudinally. This behavior is easily seen in an elastic band.

A negative Poisson ratio was found in a renormalization-group study [5] of a tethered membrane fluctuating about a planar mean conformation. This behavior can be understood [13] by imagining a crumpled sheet of paper. If one tries to pull out the folds again by applying stress in one direction, the paper will stretch in the perpendicular direction. In a subsequent study [11], it was shown that the negative Poisson ratio persists even if weak quenched disorder is included. In both cases, the negative Poisson ratio arises from the entropic properties of the network. Qualitatively speaking, the phase space which becomes available for fluctuations perpendicular to the direction of the applied stress increases with the applied stress.

More recently, we have shown that even for a membrane, fluctuations in the third dimension are not required for a negative Poisson ratio, since a strictly two- dimensional tethered membrane can exhibit this feature too [7]. This membrane consists of a regular triangular network of beads subject to a square-well interaction and an entropic tension. Again, the negative Poisson ratio is essentially an entropic effect since the elasticity of the tethers is entropic even though the tension provides a contribution to the elasticity.

The entropic origin of a negative Poisson ratio is quite different from models $[14,15]$ in which a negative Poisson ratio arises from the mechanics of a material's basic building blocks by a clever arrangement of rods, disks, and topological constraints, or from specific molecular structures [16]. Motivation for such models has been provided by the successful fabrication of foams [17] and polymeric materials [14] which indeed show a negative Poisson ratio.

In the present work we use mean-field approximations and computer simulations of several different twodimensional networks to elucidate the role which energetic, entropic, and topological factors play in the formation of a negative Poisson ratio. Our models have their origin in the "tethered network" model of Kantor, Kardar, and Nelson $[4,18]$, in which a membrane is represented by a triangulated network of $N$ vertices and $3 N$ bonds. A given vertex has sixfold coordination to a fixed set of neighboring vertices, although the length and orientation of each bond between vertices can change.

Here we investigate four different systems, each of which has (i) either a square well or Hooke's law for the bond potential energy and (ii) either bond-crossing allowed or bond-crossing forbidden. In the square-well models, the interaction is infinitely repulsive for distances larger than a cutoff length. Our square-well networks have a vanishing minimum distance between vertices, so it is somewhat different from Refs. $[4,18]$ where the minimum distance is finite. For Hooke's-law springs, there is no maximum cutoff length. The origin of the 
elasticity in the square-well and Hooke's-law networks could be described as entropic and energetic, respectively. Bond crossing (ii) implies that the network can penetrate itself (or form a pleat) similar to the self-penetrating phantom membrane in three dimensions $[4,18]$. With no bond-crossing allowed, the membrane is self-avoiding. These two types of networks allow us to probe a topology dependence of the negative Poisson ratio.

The simulations are performed at finite tension [19]. For highly stretched membranes bond crossing is unimportant. However, at moderate tension, bonds may cross frequently. Figure 1 shows two configurations from the square-well simulations. Both simulations are performed at the same tension and drawn at the same scale. In Fig. 1(a), bond crossing is forbidden while in Fig. 1(b) it is allowed. Close inspection of Fig. 1(b) shows many situations in which there are crossed bonds. As will be shown, at small tensions bond crossing has a strong effect on membrane behavior.

We investigate the elastic properties of these networks for all four combinations of (i) and (ii) using mean-field approximations and computer simulations. We find that

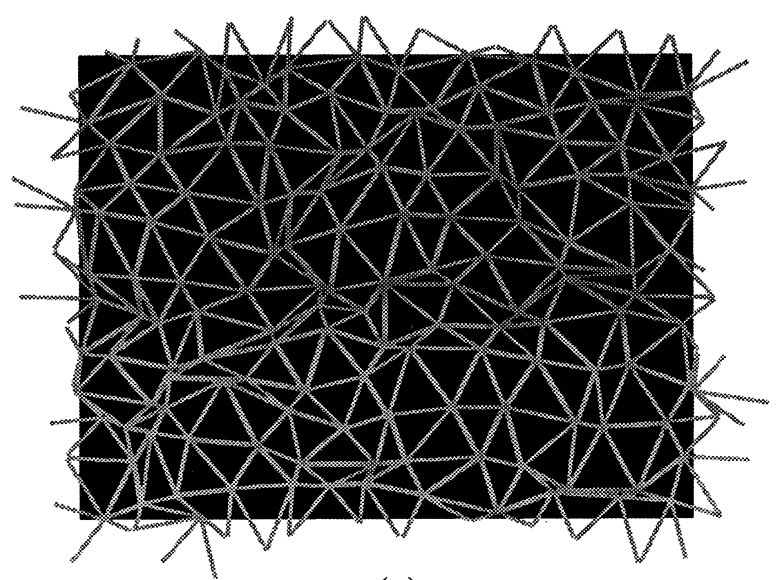

(a)

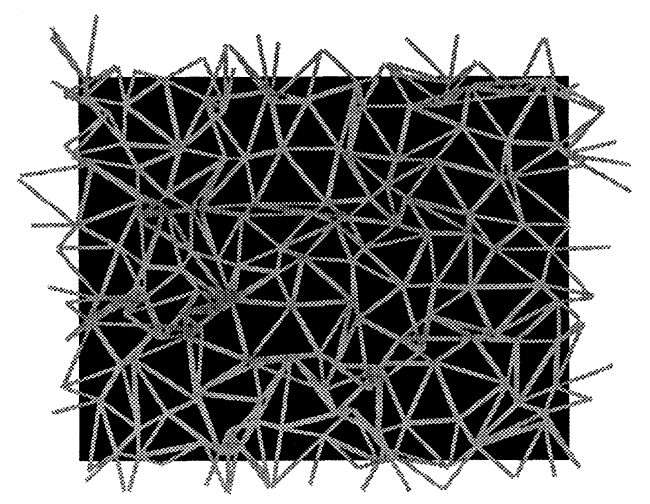

(b)

FIG. 1. Sample configurations for square-well potential interactions. Part (a) is a self-avoiding network while part (b) is not self-avoiding. Both networks were simulated at $\beta P s_{\max }^{2}=-4$, have $N=144$ vertices, and are drawn to the same scale. some of the properties are universal. In particular, the negative value of the Poisson ratio appears in at least part of the parameter space for all networks. On the other hand, we find that a phase transition to a collapsed state is specific to phantom membranes.

The paper is organized as follows. We first recall the definitions of the elastic constants in Sec. II. Because of its simplicity and use in many three-dimensional investigations, the square-well potential is investigated first in Sec. III. Next, the Hooke's-law network is studied in Sec. IV. Each of these sections begins with a mean-field approach to the networks to gain intuition about their behavior, followed by the full simulation results. Phantom networks of either interaction potential are observed to collapse at small tensions, and an estimate of the collapse tension is given in Sec. V. Our conclusions are summarized in Sec. VI. Details of the computational techniques are given in the Appendixes.

\section{ELASTIC CONSTANTS AND POISSON RATIO}

To put the discussion of elasticity on a mathematical footing, consider a continuum model for a membrane confined to a two-dimensional plane. This membrane is described by two lateral displacement fields $u_{1}$ and $u_{2}$, which are functions of the reference coordinate system $\left(x_{1}, x_{2}\right)$ attached to the membrane. The strain tensor for this membrane is defined in terms of the displacement fields by

$$
u_{i j}=\left[\frac{\partial u_{i}}{\partial x_{j}}+\frac{\partial u_{j}}{\partial x_{i}}\right] / 2,
$$

where the indices $i$ and $j$ have values of 1 and 2 . The general expression for the elastic energy in terms of the strain tensor is [20]

$$
H_{\mathrm{el}}=\int d^{2} x\left[(\lambda / 2)(\operatorname{Tr} u)^{2}+\mu \sum_{i, j} u_{i j}^{2}\right],
$$

where $\mu$ and $\lambda$ are called the Lamé coefficients. Equation (2) can be rewritten as

$$
\begin{aligned}
H_{\mathrm{el}}=\int d^{2} x\{ & K_{A}\left(u_{11}+u_{22}\right)^{2} / 2 \\
& \left.+\mu\left[\left(u_{11}-u_{22}\right)^{2} / 2+2 u_{12}^{2}\right]\right\},
\end{aligned}
$$

where $K_{A}$ and $\mu$ are the area compressibility and shear modulus, respectively. In two dimensions, $\lambda$ and the Poisson ratio $\sigma$ are related to these elastic constants through

$$
\begin{aligned}
& \lambda=K_{A}-\mu, \\
& \sigma=\left(K_{A}-\mu\right) /\left(K_{A}+\mu\right) .
\end{aligned}
$$

While the bulk and shear moduli are required to be positive for mechanically stable systems, there is no such condition on $\lambda$ and $\sigma$. Physically, a system with negative $\sigma$ expands transversely when stretched longitudinally.

\section{SQUARE-WELL INTERACTION}

The first network which we investigate has a squarewell (SW) potential governing the bond energies. We 
choose a potential which is infinitely repulsive for bond length $x$ larger than a cutoff length $s_{\max }$ and zero otherwise:

$$
V_{\mathrm{SW}}(x)=\left\{\begin{array}{l}
0 \text { for } 0 \leq x \leq s_{\max } \\
+\infty \text { for } x>s_{\max }
\end{array} .\right.
$$

Thus the membrane area per vertex cannot exceed $\left(3^{1 / 2} / 2\right) s_{\max }^{2}$. The membrane is also subject to a negative pressure or tension $P<0$, which enters as the dimensionless quantity $\beta P s_{\max }^{2}$, where $\beta$ is the inverse temperature.

\section{A. Mean-field expectations}

To gain some intuition about the elastic properties of the square-well network, we use a crude mean-field argument in which we ignore correlations between the different vertices. Then the physics of the network can be derived from that of a single vertex. Recalling from Fig. 1 that an elementary triangle of the network contains half a vertex, we take a parallelogram (rhomboid) to be the "unit cell" associated with a single vertex. We fix one side of the parallelogram to lie along the positive $x$ axis and have length $s_{1}$. The other side has length $s_{2}$ and makes an angle $\phi$ with the $x$ axis. The square-well potential restricts the variables to lie in the range $0 \leq s_{1} \leq s_{\max }$, $0 \leq s_{2} \leq s_{\max }$, and $0 \leq \phi \leq \phi_{0}$, where the angle $\phi_{0}$ is given by

$$
\begin{aligned}
\phi_{0}= & \begin{array}{l}
\pi \text { for } 0 \leq\left(s_{1}+s_{2}\right) / s_{\max } \leq 1 \\
\arccos \left[\left(s_{1}^{2}+s_{2}^{2}-s_{\max }^{2}\right) /\left(2 s_{1} s_{2}\right)\right]
\end{array} \\
& \text { for } 1<\left(s_{1}+s_{2}\right) / s_{\max } \leq 2 .
\end{aligned}
$$

Equation (7b) arises from the network triangle constraint that the diagonal opposite to the angle $\phi$ must have a length less than $s_{\max }$.

The partition function for the single vertex then reads

$$
Z=\int d s_{1} \int d s_{2} s_{2} \int d \phi \exp \left[-\beta P s_{1} s_{2} \sin (\phi)\right],
$$

where $\beta$ is the inverse temperature and $P<0$ is the pressure, taken to be negative here to represent a tension. Since the area $a_{v}$ belonging to the vertex is given by $s_{1} s_{2} \sin (\phi)$, the mean area of the vertex $\left\langle a_{v}\right\rangle$ can be calculated as

$$
\begin{aligned}
\left\langle a_{v}\right\rangle=\int d s_{1} \int d s_{2} s_{2} \int & d \phi s_{1} s_{2} \sin (\phi) \\
& \times \exp \left[-\beta P s_{1} s_{2} \sin (\phi)\right] / Z .
\end{aligned}
$$

An analogous expression holds for the mean value $\langle f\rangle$ of any function $f\left(s_{1}, s_{2}, \phi\right)$. For a large number $N$ of these vertices, the total mean area $\langle A\rangle$ becomes

$$
\langle A\rangle=N\left\langle a_{v}\right\rangle
$$

with a variance of

$$
\left\langle A^{2}\right\rangle-\langle A\rangle^{2}=N\left(\left\langle a_{v}^{2}\right\rangle-\left\langle a_{v}\right\rangle^{2}\right)
$$

from the central limit theorem. Hence an approximation to the compressibility modulus of the network is just

$$
K_{A}=\langle A\rangle /\left(\left\langle A^{2}\right\rangle-\langle A\rangle^{2}\right)=\left\langle a_{v}\right\rangle /\left(\left\langle a_{v}^{2}\right\rangle-\left\langle a_{v}\right\rangle^{2}\right) .
$$

The shear modulus $\mu$ is determined through Young's moduli which can be obtained from the fluctuations in the $x$ and $y$ directions as described in Appendix B.

The results for $K_{A}, \mu$ and $\lambda=K_{A}-\mu$, obtained from the mean-field argument, are shown in Fig. 2. One can see that at large tension (negative pressure), the compression modulus substantially exceeds the shear modulus and the Lamé coefficient $\lambda$ is positive as is the Poisson ratio. In fact, as the tension becomes very large, the ratio of $K_{A} / \mu$ approaches the limiting value of 2 expected for a harmonic network [4]. At more modest tension, the compression modulus decreases compared to the shear modulus, and finally at a critical pressure of about $\beta P_{c} s_{\max }^{2}=-7, \lambda$ and $\sigma$ become negative. The Poisson ratio is observed to be negative over the range of tensions $0 \leq-P \leq-P_{c}$ in the mean-field model.

\section{B. Monte Carlo simulations}

The details of the simulation are summarized in the Appendixes. Essentially, a rectangular "patch" of the membrane with periodic boundary conditions is simulated using standard Monte Carlo techniques for $N=144$ vertices. The elastic constants are determined by the fluctuations in the membrane size. The statistical uncertainties are in the $10 \%$ range for the elastic constants.

In this section, we discuss and compare results from the self-avoiding and phantom membranes. The most obvious difference in the behavior of these networks can be seen in the area per vertex $\langle A\rangle / N$. This is shown in Fig. 3 , which also shows the area per vertex from the simple mean-field calculation. First, one sees that for tensions

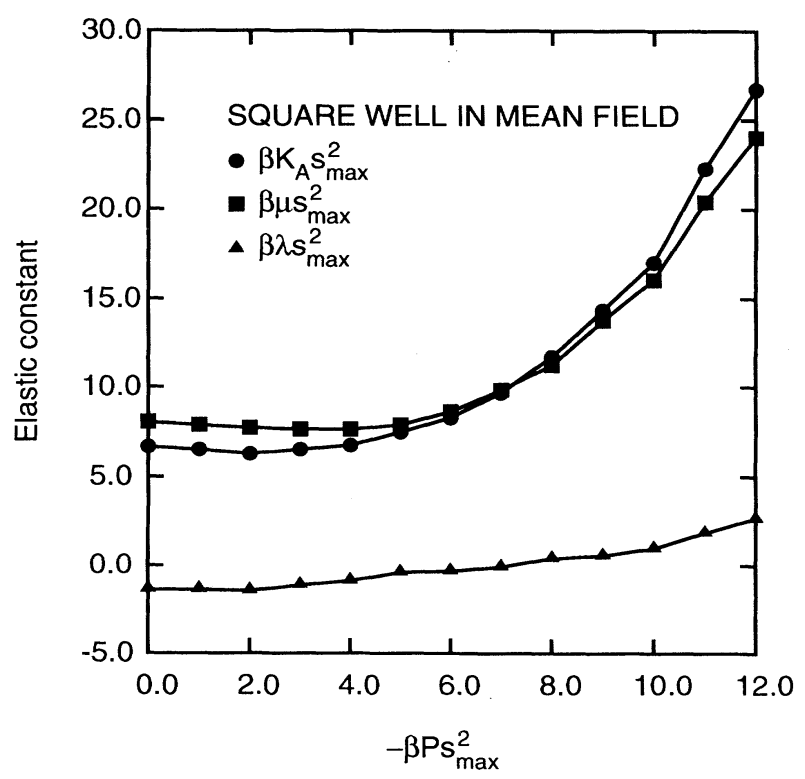

FIG. 2. Elastic constants $\beta K_{A} s_{\max }^{2}, \beta \mu s_{\max }^{2}$, and $\beta \lambda s_{\max }^{2}$ obtained from a mean-field approximation to the square-well network shown as a function of $\beta P s_{\max }^{2}$. 


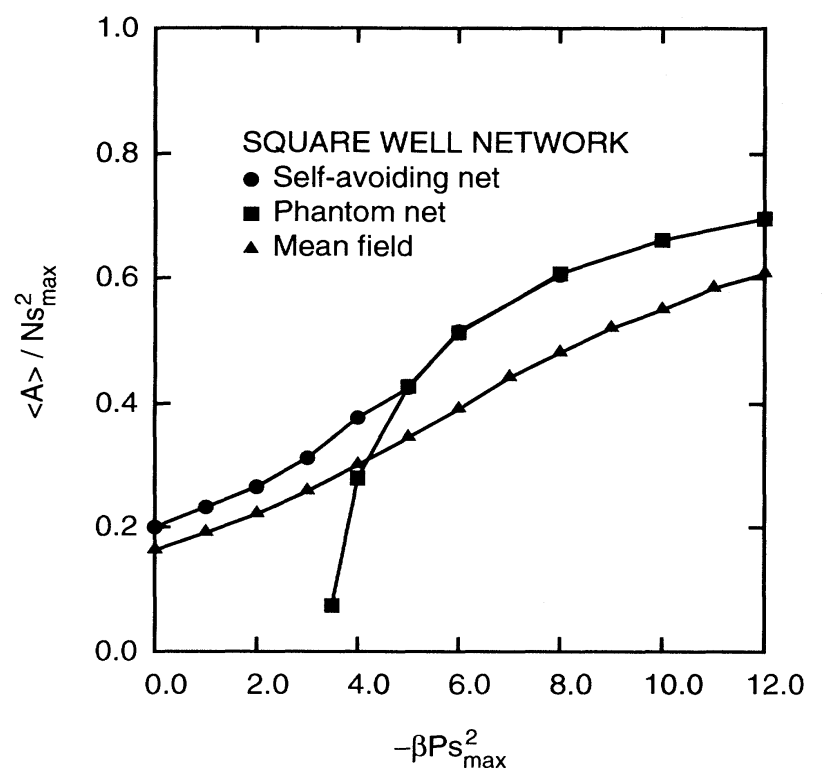

FIG. 3. Normalized area per vertex $\langle A\rangle / N s_{\max }^{2}$ as a function of tension $\beta P s_{\max }^{2}$ for the square-well network. The figure shows results for both the self-avoiding and phantom membranes as well as the mean-field estimate. Estimated uncertainties are approximately $1 \%$.

greater than $-\beta P s_{\max }^{2}>5$, the areas of the two networks are essentially the same. This is to be expected as the networks approach the large area limit of $A / N s_{\max }^{2}=\frac{1}{2} \sqrt{3}$, where the bonds cannot intersect and the self-avoidance issue is irrelevant. It is also interesting to note that the mean-field expression for the area is a reasonable approximation to the full self-avoiding network. The mean-field result parallels the full network, but is typically about $10-15 \%$ lower.

The most interesting feature of Fig. 3 is the collapse of phantom membranes at $-\beta P s_{\max }^{2}<3.4$. This behavior is similar to that of phantom membranes with free boundary conditions embedded in three dimensions [18]. From Fig. 3, the collapse transition appears to be second order. The reason for the collapse is the large entropy gain which the network receives from the configuration space available when bond crossing is allowed. As a technical point, the simulation data near the collapse transition are taken with $N=576$ because the intervertex separations conflict with the small values of $L_{x}$ and $L_{y}$ in the periodic boundary conditions, making the $N=144$ data unreliable.

The behavior of the shear modulus $\beta \mu s_{\max }^{2}$ is shown as a function of the normalized area per vertex $\langle A\rangle / N s_{\text {max }}^{2}$ in Fig. 4. Over much of the range investigated, both networks show the same shear modulus at equivalent areas, although not at equivalent pressures. As one would expect, as the membrane becomes less stretched, fluctuations in the intervertex separation decrease the shear (and compression) modulus. Since at larger tension, where the membrane is highly stretched, all triangles have a similar (equilateral) shape, the mean field does a good job in representing the shear modulus in this regime. At low tension, there is a greater variety of single triangle shapes

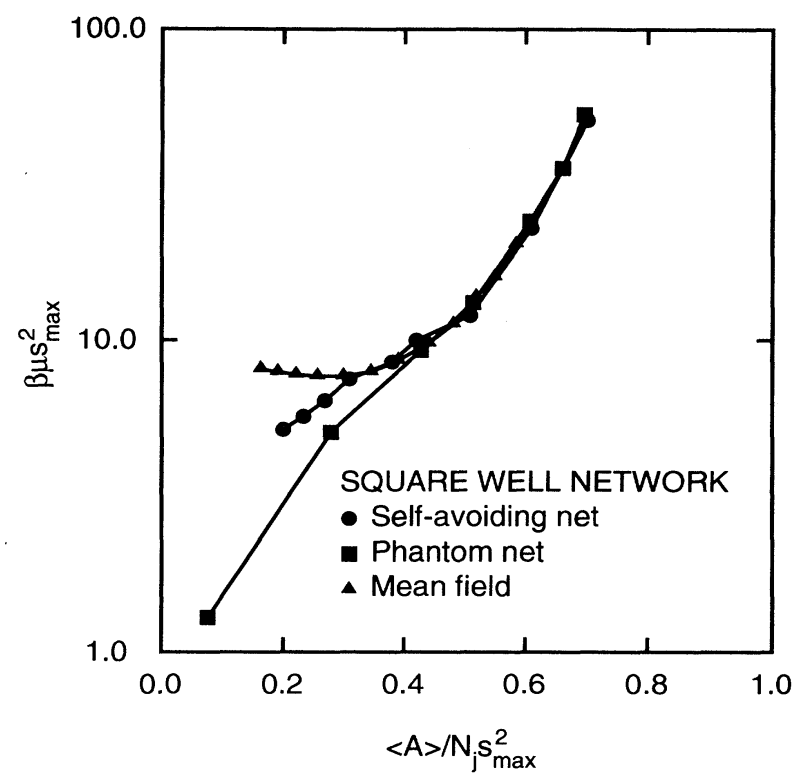

FIG. 4. Shear modulus $\beta \mu s_{\max }^{2}$ as a function of normalized area per vertex $\langle A\rangle / N s_{\max }^{2}$ for the square-well network. The figure shows results for both the self-avoiding and phantom membranes as well as the mean-field estimate.

(see Fig. 1) and the agreement is not as good.

Finally, in Fig. 5 we show the Poisson ratio $\sigma$. For both self-avoiding and phantom networks there is a range of areas (or tensions) for which $\sigma$ is negative. At very large tension, we expect $\sigma$ to be positive, since in the harmonic limit we should have $K_{A}=2 \mu$ or $\sigma=\frac{1}{3}$. Similarly,

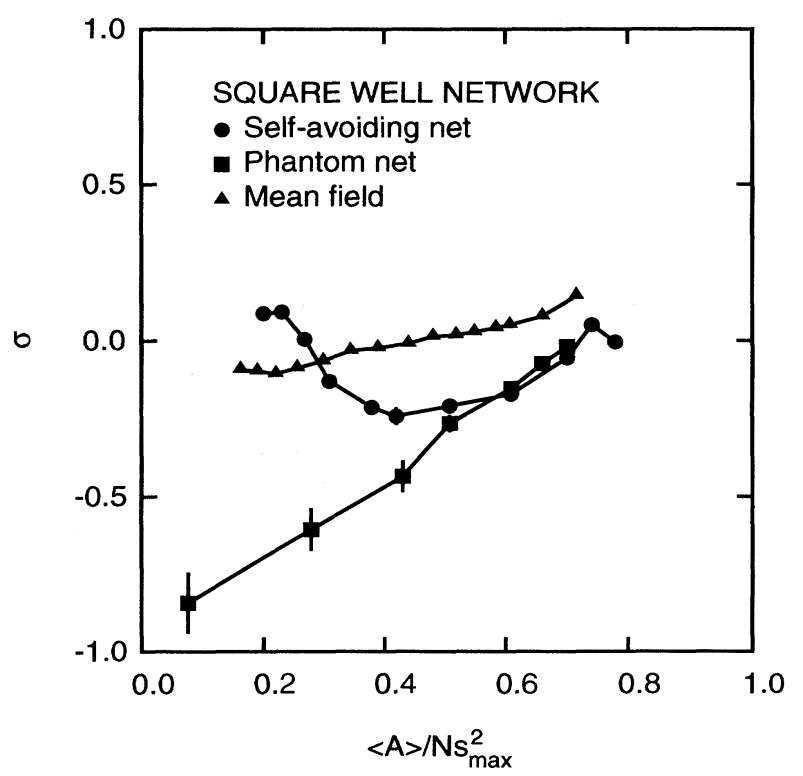

FIG. 5. Poisson ratio $\sigma$ as a function of normalized area per vertex $\langle A\rangle / N s_{\max }^{2}$ for the square-well network. The figure shows results for both the self-avoiding and phantom membranes as well as the mean-field estimate. 
at positive pressure where the networks behave more like ideal gases, $\sigma$ should also be positive. It is only in the intermediate tension regime where negative values are obtained. The mean field also predicts negative values for $\sigma$ over a range in tension which roughly corresponds to the values obtained in the simulation. We note that both the self-avoiding and phantom networks show the same value for $\sigma$ if the network area exceeds about $60 \%$ of its fully stretched area.

\section{HOOKE'S-LAW INTERACTION}

An alternative network which we can use to explore the domain of negative Poisson ratio is a network of Hooke's-law (HL) springs. We parametrize the Hooke's law potential as

$$
V_{\mathrm{HL}}=(k / 2)\left(s-s_{0}\right)^{2},
$$

where $k$ is the spring constant and $s_{0}$ is the equilibrium length. Here, there are two length scales in the problem: $s_{0}$ and $(\beta k)^{-1 / 2}$, where $\beta$ is the inverse temperature. We choose to absorb $k$ into the units, so $s_{0}$ is quoted in units of $(\beta k)^{-1 / 2}$ and a dimensionless tension is $P / k<0$.

\section{A. Mean-field estimates}

Since the elasticity of the Hooke's law network is of energetic origin, a mean-field estimate of its elastic constants can be obtained by calculating the energy of particular deformations around an equilibrium conformation of equilateral triangles. The equilibrium conformation at a given pressure follows from minimizing the free energy per vertex $f_{v}$,

$$
f_{v}=(k / 2)\left\{3\left(s-s_{0}\right)^{2}+3^{1 / 2} P s^{2} / k\right\} \text {. }
$$

At fixed pressure, the equilibrium spring length $s_{p}$ is

$$
s_{p}=s_{0} /\left(1+P / 3^{1 / 2} k\right),
$$

from which the equilibrium area per vertex is

$$
\left\langle a_{v}\right\rangle=\left(3^{1 / 2} / 2\right) s_{p}^{2}=\left(3^{1 / 2} / 2\right) s_{0}^{2} /\left(1+P / 3^{1 / 2} k\right)^{2} \text {. }
$$

This expression shows us that the area expands without bound when $P / k<-3^{1 / 2}$, independent of the value of $s_{0}$. Thus the mean-field network is unstable for $P / k<-3^{1 / 2}$, a characteristic of all the Hooke's-law simulations which we report.

Consider now the situation in which there is a uniform stretching about $s_{p}$, say $s=(1+\epsilon) s_{p}$, where $\epsilon$ is small. Then the change in the free energy per vertex associated with this deformation is

$$
\Delta f_{v}=(3 k / 2) \epsilon^{2} s_{p}^{2}\left(1+P / 3^{1 / 2} k\right)
$$

We divide this expression by the area per vertex $\left\langle a_{v}\right\rangle$ to obtain the change in energy density, which can be compared with the expression $\Delta H_{\mathrm{el}}=2 K_{A} \epsilon^{2}$ for this deformation from Eq. (3). Thus we predict that

$$
K_{A} / k=\left(3^{1 / 2} / 2\right)\left\{1+P /\left(3^{1 / 2} k\right)\right\} \text {. }
$$

Note that the compression modulus vanishes at
$P / k=-3^{1 / 2}$, as expected from the instability predicted by Eq. (16).

The shear modulus $\mu$ can be obtained by considering an area-preserving displacement of one of the vertices in a direction parallel to the side of the mean-field triangle opposite the vertex. To be specific, suppose that the base of the mean-field equilateral triangle with length $s_{p}$ lies along the $x$ axis. If we displace the vertex opposite the base by an amount $\epsilon s_{p}$ in the $x$ direction, then the meanfield free energy per vertex changes by

$$
\Delta f_{v}=k \epsilon^{2} s_{p}^{2}\left\{1-\frac{3}{4}\left(s_{0} / s_{p}\right)\right\} .
$$

Now the diagonal elements of the strain tensor for this deformation vanish, and the off-diagonal elements obtained from Eq. (1), are $u_{12}=u_{21}=\epsilon / 3^{1 / 2}$. Hence the change in the elastic free energy density is $\Delta H_{\mathrm{el}}=2 \mu \epsilon^{2} / 3$. Eliminating $s_{0}$ from Eq. (19) and dividing it by the area per vertex, we find that the shear modulus is

$$
\mu / k=\left(3^{1 / 2} / 4\right)\left(1-3^{1 / 2} P / k\right) \text {. }
$$

Finally, Eqs. (18) and (20) yield

$$
\begin{aligned}
& \lambda / k=\left(3^{1 / 2} / 4\right)\left\{1+5 P /\left(3^{1 / 2} k\right)\right\}, \\
& \sigma=\left\{1+5 P /\left(3^{1 / 2} k\right)\right\} /\left\{3-P /\left(3^{1 / 2} k\right)\right\} .
\end{aligned}
$$

Equations (18), (20), and (21) reduce to the harmonic network expressions at zero tension, as one would expect. These simple expressions for $K_{A}, \mu$ and $\lambda$ are plotted in Fig. 6 for tension $P / k<0$. Note that all of these expressions are independent of $s_{0}$. As with the square-well network, there is a region of the tension $3^{1 / 2} / 5<-P / k$ $<3^{1 / 2}$ over which $\lambda$ and $\sigma$ are negative.

We expect this mean-field estimate to be reliable when the fluctuations in the spring length are small compared

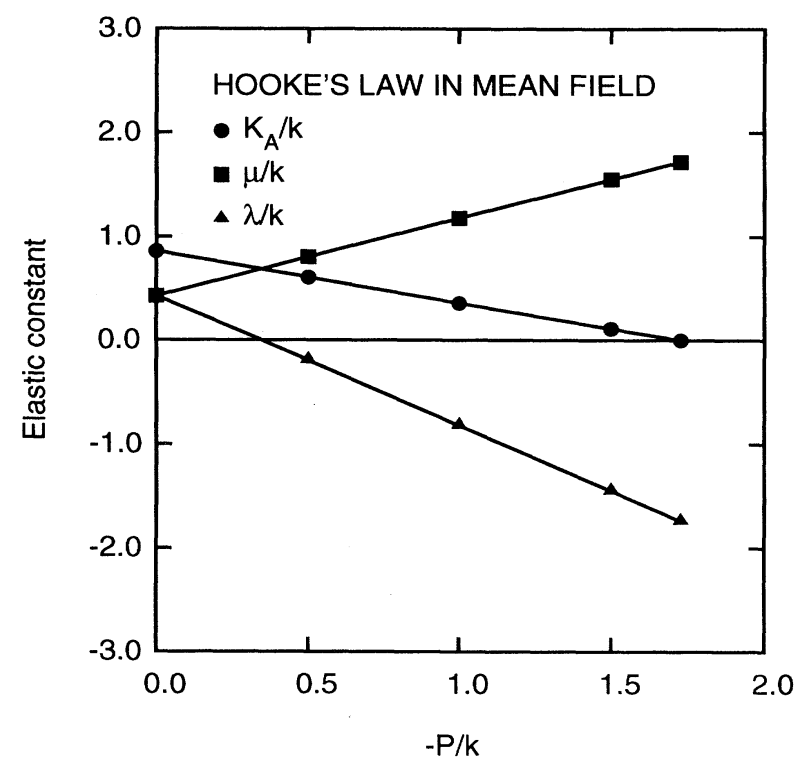

FIG. 6. Elastic constants $K_{A} / k, \mu / k$, and $\lambda / k$ obtained from a mean-field approximation to a Hooke's-law network, shown as a function of tension $P / k$. In this mean-field approximation, the elastic constants are independent of spring length $s_{0}$. 
to the equilibrium length, i.e., $\left\langle\left(s-s_{p}\right)^{2}\right\rangle<s_{p}^{2}$. Using Eqs. (14) and (15), this criterion becomes

$$
s_{0} /(\beta k)^{1 / 2} \gg\left(1+P / 3^{1 / 2} k\right) / 3^{1 / 2} \text {. }
$$

\section{B. Monte Carlo simulations}

Our simulations for the Hooke's-law network are performed in the same way as the square-well network of Sec. III. However, the Hooke's-law parameter space is much larger because we examine the dependence of the elastic properties on the equilibrium length $s_{0}$ as well as on the tension. We first discuss self-avoiding networks and then report a more limited set of simulations with phantom networks. Throughout this section, the basic length unit is $(\beta k)^{-1 / 2}$.

In the mean-field approach outlined in Sec. IV $A$, the area per vertex at zero tension is $\left(3^{1 / 2} / 2\right) s_{0}^{2}$, a quantity which we define as $a_{0}$. The mean-field prediction of Eq. (16) can then be rewritten as

$$
\langle A\rangle / N a_{0}=\left(1+P / 3^{1 / 2} k\right)^{-2} .
$$

This expression is compared with the full simulation for self-avoiding networks in Fig. 7. One can see that the simulations rapidly converge to the mean-field results at $s_{0}=1$. We have not shown the area of networks at $s_{0}>1$ since they are almost identical to the mean-field results. The figure also shows that the area expands dramatically as $-P / k$ approaches $3^{1 / 2}$, as expected from the meanfield approximation.

The behavior of the compression modulus is shown in Fig. 8 for self-avoiding networks. Again, the agreement

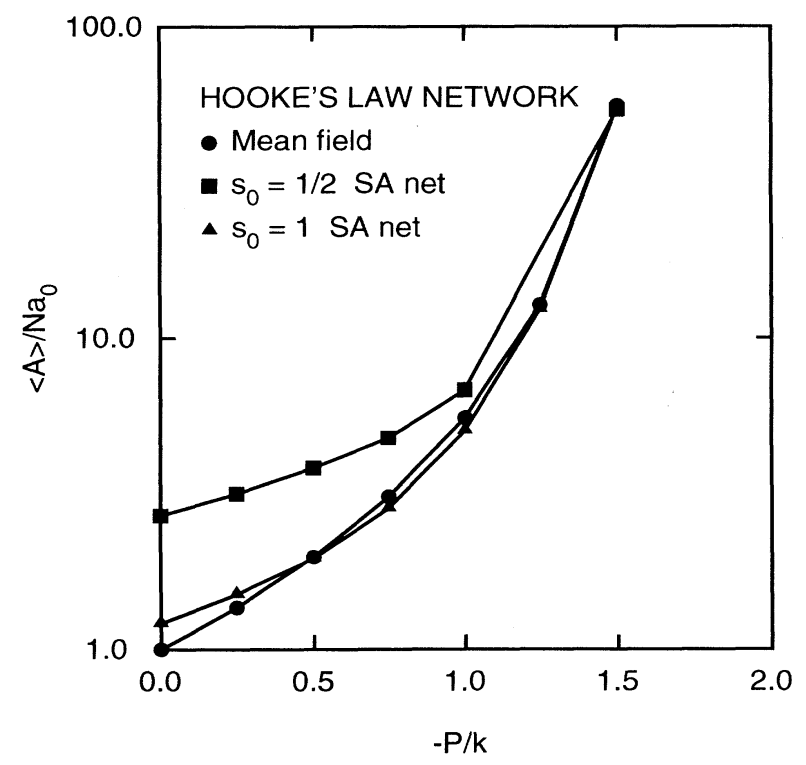

FIG. 7. Normalized area per vertex $\langle A\rangle / N a_{0}$ as a function of tension $P / k$ for the Hooke's-law network. The figure shows the mean-field prediction and results for the self-avoiding (SA) network with equilibrium spring length $s_{0}=\frac{1}{2}$ and 1 in units of $(\beta k)^{-1 / 2}$. Estimated uncertainties are approximately $1 \%$.

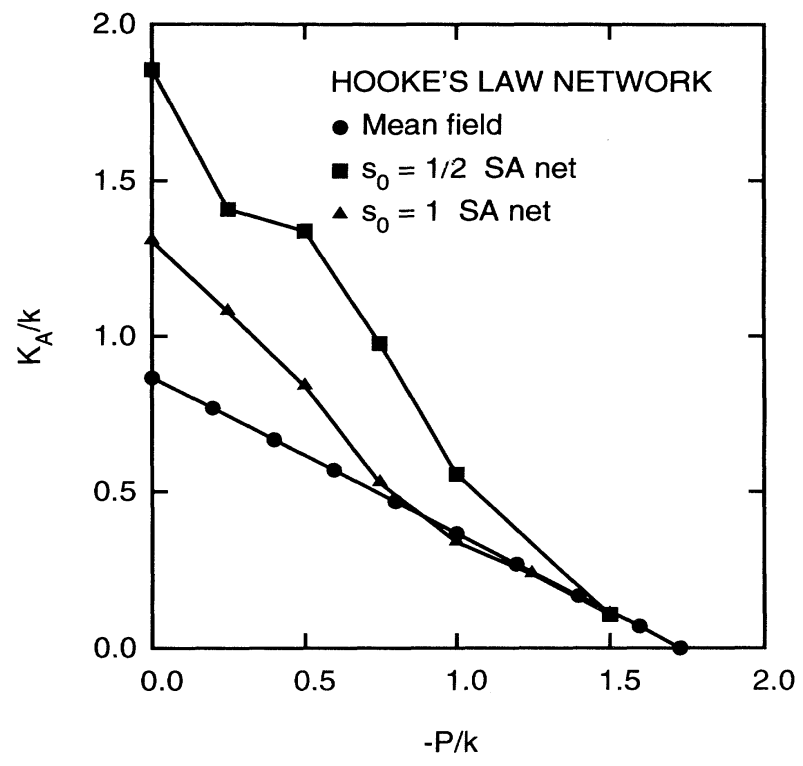

FIG. 8. Compression modulus $K_{A} / k$ as a function of tension $P / k$ for the Hooke's-law network. The figure shows the meanfield estimate and results for the self-avoiding network with equilibrium spring length $s_{0}=\frac{1}{2}$ and 1 in units of $(\beta k)^{-1 / 2}$.

with the mean-field predictions improves with increasing $s_{0}$ and $-P / k$ exactly as the criterion of Eq. (22) predicts. However, one must go to much larger $s_{0}$ before the agreement becomes as good as is observed for the area in Fig. 7. The simulations show that the networks become more difficult to compress the smaller the elementary triangle size becomes. This can be seen either by varying $s_{0}$ at fixed $P / k$ (most obvious at $P / k=0$ ) or by varying $P / k$ at fixed equilibrium length $s_{0}$.

The shear modulus is shown in Fig. 9 for self-avoiding Hooke's-law networks. As with Figs. 7 and 8, there is a discrepancy between the simulation and the mean-field results at small $s_{0}$. However, the disagreement is reduced as the spring length increases and the agreement is very good for $s_{0}=4$ over the range of tensions investigated.

The Poisson ratio obtained from the shear and compression moduli is shown in Fig. 10. Here we find very little change of $\sigma$ with spring length except for very small tensions. Further, the agreement with the meanfield calculation is excellent over most of the pressure range. As with the square-well interaction, there is a substantial tension range for which $\sigma$ and $\lambda$ are negative.

All of the Hooke's-law simulations reported thus far in this section are for self-avoiding (SA) networks. If we drop the self-avoidance constraint, then the network behavior changes radically for small tensions, just as it does for phantom square-well networks. We begin our discussion of self-avoidance effects by examining the area per vertex, shown in Fig. 11. A rapid decrease in area is observed for the phantom membrane as $-P / k$ is reduced. For the networks shown with $s_{0}=1$, at a tension of around $-P / k=0.6$ the membrane becomes very highly overlapping, with the area per vertex appearing to go to zero. The transition tension depends on $s_{0}$, and is 


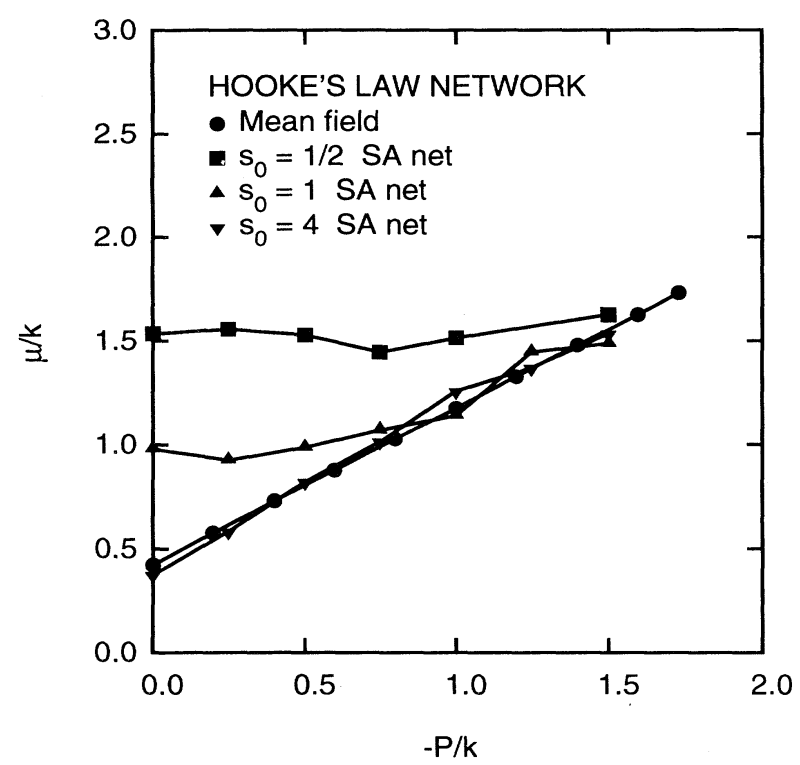

FIG. 9. Shear modulus $\mu / k$ as a function of tension $P / k$ for the Hooke's-law network. The figure shows the mean-field estimate and results for the self-avoiding network with equilibrium spring length $s_{0}=\frac{1}{2}, 1$, and 4 in units of $(\beta k)^{-1 / 2}$.

larger (i.e., $P / k$ is more negative) for smaller values of $s_{0}$. For $s_{0}$ near zero, the membrane collapses as soon as the tension is reduced only slightly from $-3^{1 / 2}$. At the other extreme, networks with $s_{0}=4$ collapse only near zero tension.

The behavior of the elastic constants in the phantom network also deviates from the self-avoiding network

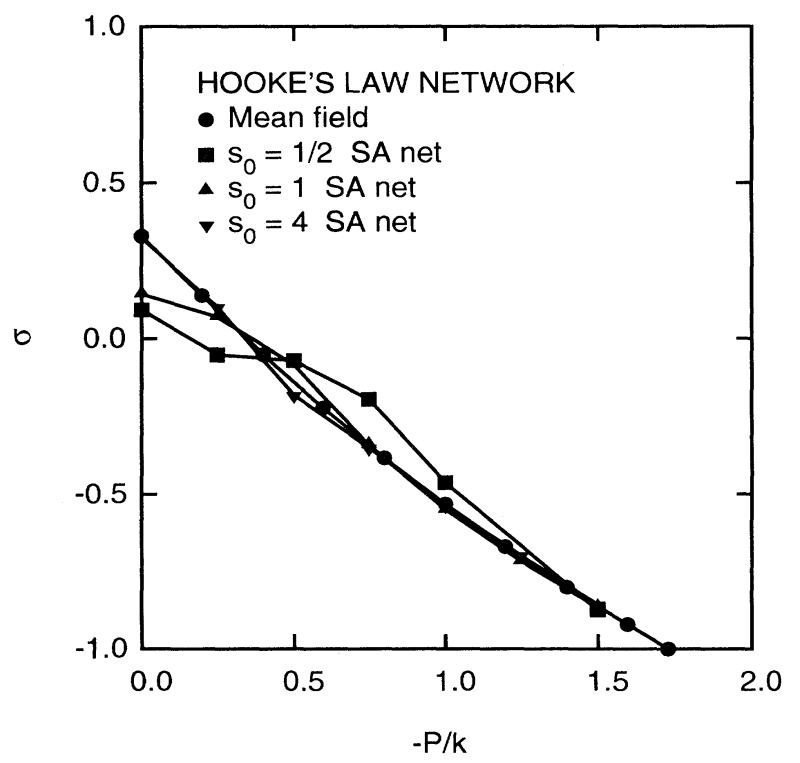

FIG. 10. Poisson ratio $\sigma$ as a function of tension $P / k$ for the Hooke's-law network. The figure shows the mean-field estimate and results for the self-avoiding network with equilibrium spring length $s_{0}=\frac{1}{2}, 1$, and 4 in units of $(\beta k)^{-1 / 2}$.

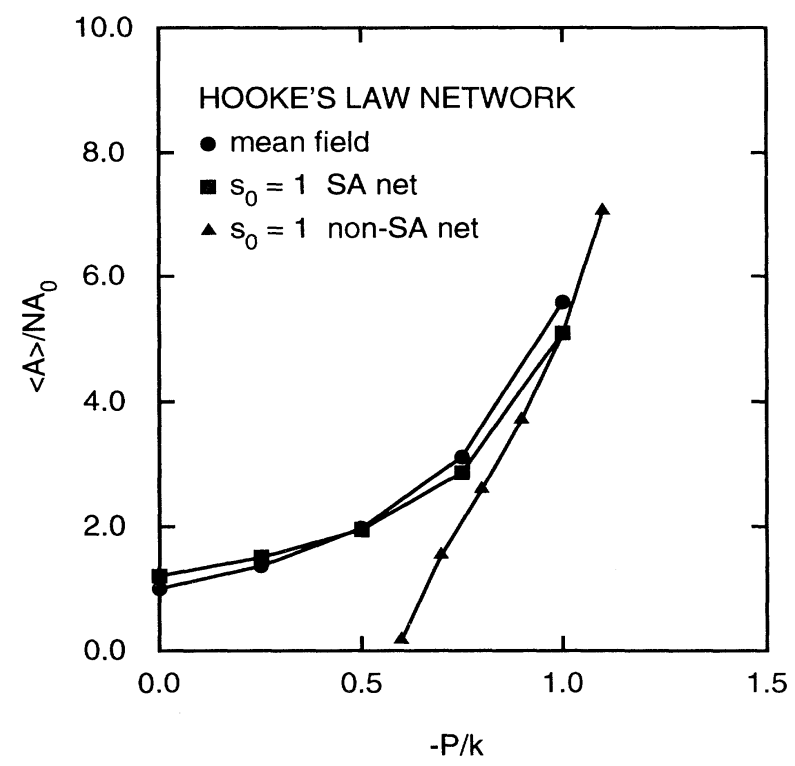

FIG. 11. Normalized area per vertex $\langle A\rangle / N a_{0}$ as a function of tension $P / k$ for the Hooke's-law network. The figure shows the mean-field prediction and results for the self-avoiding (SA) and phantom (non-SA) networks with equilibrium spring length $s_{0}=1$ in units of $(\beta k)^{-1 / 2}$. Estimated uncertainties are approximately $1 \%$.

near the collapse tension. Examples of this are shown in Figs. 12 and 13 for phantom and SA networks with $s_{0}=1$. However, as Fig. 13 emphasizes, the negative Poisson ratio is not restricted to self-avoiding membranes, and the phantom network has negative $\sigma$ over its entire stability range.

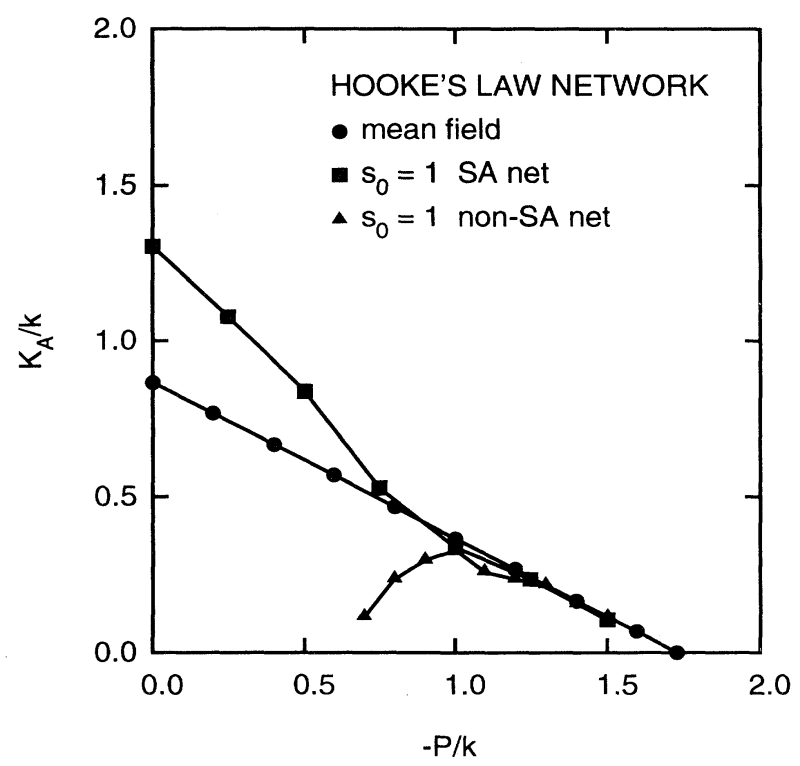

FIG. 12. Compression modulus $K_{A} / k$ as a function of tension $P / k$ for the Hooke's-law network. The figure shows the mean-field prediction and results for the self-avoiding (SA) and phantom (non-SA) networks with equilibrium spring length $s_{0}=1$ in units of $(\beta k)^{-1 / 2}$. 


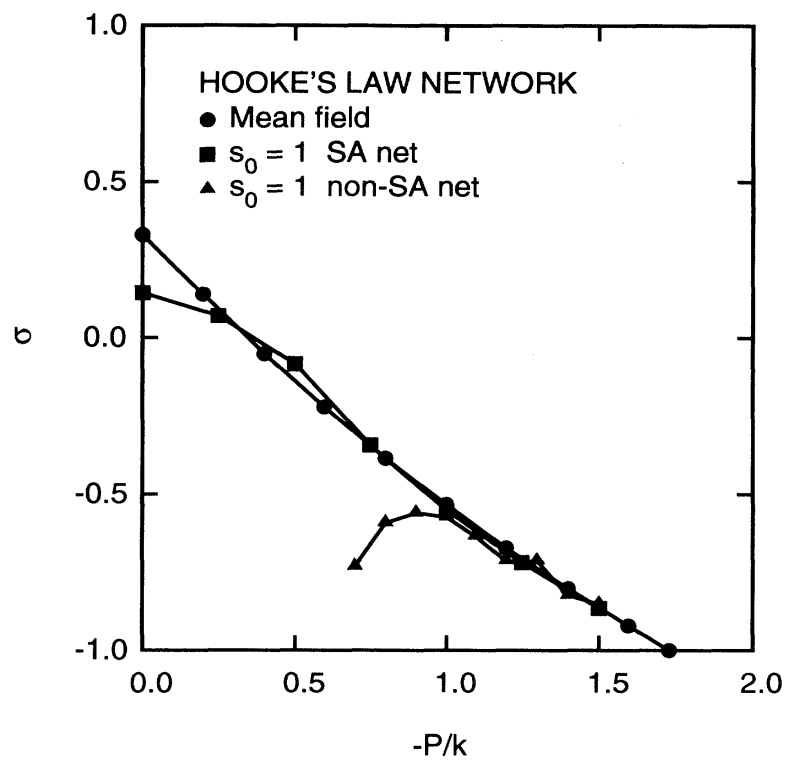

FIG. 13. Poisson ratio $\sigma$ as a function of tension $P / k$ for the Hooke's-law network. The figure shows the mean-field prediction and results for the self-avoiding (SA) and phantom (nonSA) networks with equilibrium spring length $s_{0}=1$ in units of $(\beta k)^{-1 / 2}$.

\section{PHANTOM-MEMBRANE COLLAPSE}

Both the square-well and Hooke's-law phantom membranes collapse to a highly compact form at small tensions. This type of collapse is reminiscent of phantom polymerized membranes in three embedding dimensions. In this section, we present an argument for why such a collapse should occur and we provide a coarse estimate of the tension at the collapse transition.

It can be seen from comparing Figs. 1(a) and 1(b) that domains of bond crossing appear to occur in lines (rather than be isolated or occur in patches) in phantom squarewell networks. We show in Fig. 14 sample configurations of phantom Hooke's-law networks with $s_{0}=1$ where this phenomenon also is present. In Fig. 14(a), the tension of $-P / k=0.8$ keeps the membrane well stretched while in Fig. 14 (b) the tension of $-P / k=0.7$ is close to the collapse point (about $-P / k=0.6$ ). The regions where elementary triangles have small area or are folded over on themselves are clearly chainlike.

Figures 1 and 14 suggest that the networks resemble domains of average area triangles separated by boundaries of zero area or reversed triangles. Let us formulate a model for the collapse transition for the square-well network in terms of chains of zero-area triangles. Suppose there are $L$ zero-area triangles on a line or fold. As the collapse point is neared, each triangle on the fold reduces the area of the network by about half of the fully stretched area per triangle [i.e., half of $\left(3^{1 / 2} / 4\right) s_{\max }^{2}$, according to Fig. 3. A chain of $L$ triangles results in an increase of the pressure contribution to the free energy of $(L / 2)(-P / k)\left(3^{1 / 2} / 4\right) s_{\max }^{2}$.

Since there are many paths for the chain to take through the network, then the increase in the free energy

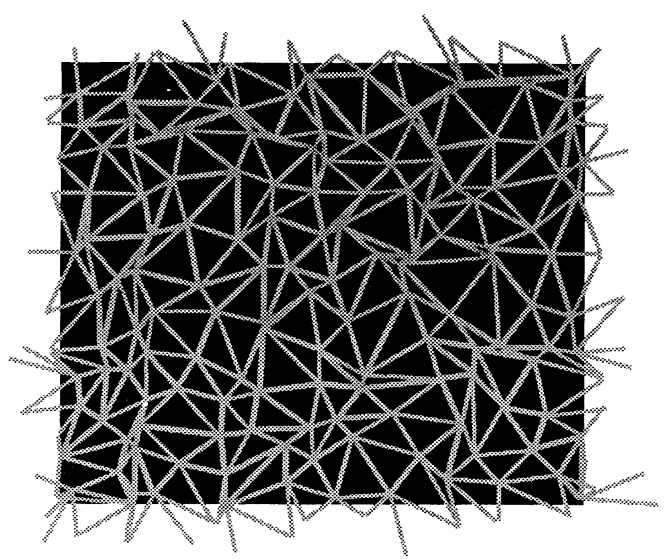

(a)

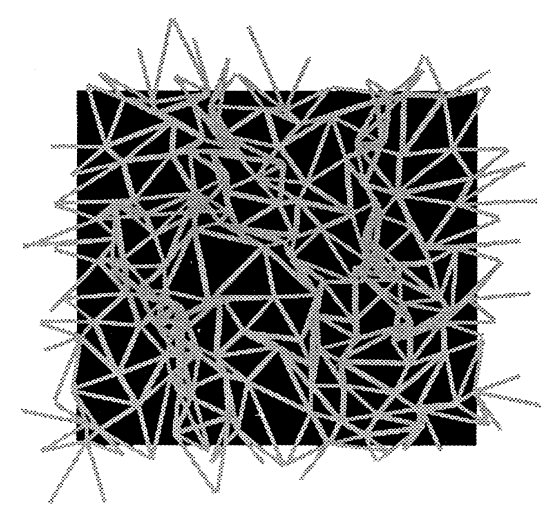

(b)

FIG. 14. Sample configurations for Hooke's-law networks. Part (a) is a self-avoiding network at $-P / k=0.8$ while part (b) is a phantom network at $-P / k=0.7$. Both networks have $N=144$ vertices and are drawn to the same scale.

from the pressure contribution is offset by a decrease in the free energy from the entropy. If the path can branch to the left or right at each triangle, then there are roughly $2^{L}$ paths for each starting point on the boundary. The entropy associated with the paths then results in a decrease in the free energy of $(L \ln 2) / \beta$, where $\beta$ is the inverse temperature. Equating the pressure and entropy contributions to the free energy gives a collapse transition tension of

$$
-\beta P s_{\max }^{2}=8 \ln 2 / 3^{1 / 2}=3.2 .
$$

That this estimate is so close to the actual transition tension of $-\beta P s_{\max }^{2}=3.4$ is entirely fortuitous, since our counting of the chain configurations and our estimates of their areas is very crude. However, the picture we obtain is that the collapse is characterized by the formation of chains of reversed or zero-area triangles.

\section{CONCLUSIONS}

We determine the elastic constants of four networks which are classified as self-avoiding or phantom and 
which have either a square-well or Hooke's-law interaction between membrane elements. All of the networks show regions of tension in which the Poisson ratio is negative. Mean-field approximations to these networks qualitatively predict their behavior and are in quantitative agreement with the full simulations for the networks at large tension. Both of the two-dimensional phantom networks which we investigate show the interesting property that they collapse at a critical value of the tension. The tension at the collapse transition can be qualitatively estimated by a model in which the collapse is characterized by chains of zero-area triangles separating domains of average-area triangles.

Our results show that one can achieve a negative Poisson ratio for two-dimensional materials without a complicated design for their components, provided the materials are subject to an isotropic tension. We find universally that $\sigma<0$ in some tension range irrespective of whether the elasticity is of energetic (Hooke's law) or entropic (square well) origin. Likewise, even phantom membranes which have fewer topological constraints show this behavior.

\section{ACKNOWLEDGMENT}

This work is supported in part by the Natural Sciences and Engineering Research Council of Canada.

\section{APPENDIX A: SIMULATION TECHNIQUE}

The two-dimensional membrane in our computational model is represented by a fixed number $(N)$ of vertices linked together by a fixed number $(3 N)$ of straight flexible bonds. The coordination of each vertex is sixfold. The minimal extension of the bonds is zero, and the maximal extension depends on the interaction potential, as described in Secs. II or III. The bonds have fixed connectivity in that their ends are always attached to the same vertices. There is no resistance to in-plane "bending" of the bonds with respect to each other.

The simulations are performed using a Metropolis Monte Carlo technique, in which an appropriately weighted sample set of configurations is generated by computer. We consider two different ways in which the vertices which define the network can move. In one case, bonds may not intersect each other, meaning that the network has no pleats and is self-avoiding. In the other case, bonds are allowed to intersect and the network is a phantom membrane. Each trial move is accepted with the Boltzmann weight $\exp (-\beta \Delta H)$, where $\Delta H$ is the energy change associated with the move. In the simulation, a sweep across the membrane involves a number of steps.

(i) All simulations. An attempt is made to change the position of each vertex by choosing a new position randomly from within a square box of length $2 l$ to the side centered on the old position. We choose $l=0.1 l_{s}$, where $l_{s}$ is the elementary length scale in the simulation and is equal to $s_{\max }$ in the square-well interaction or $(\beta k)^{-1 / 2}$ in the Hooke's-law interaction.

(ii) Self-avoiding networks. Each vertex is common to several triangles. The area of each of these triangles is equal to half of the absolute value of the cross product of the displacement vectors associated with two bonds which define the triangle. If a vertex crosses a bond during a move, then the signed area (no absolute value) changes sign. Hence self-avoidance is enforced by requiring the signed areas of all triangles associated with a vertex not change during an attempted move. The restriction is not enforced for phantom membranes.

A rectangular membrane "patch" subject to periodic boundary conditions in the $x$ and $y$ directions is used in the simulations. An isobaric simulation is performed by allowing the rectangle lengths $L_{x}$ and $L_{y}$ to vary independently; that is, the ensemble is $N P T$ rather than $N A T$. There is one trial move to rescale the rectangle size per sweep. The rescaling moves are accepted with a pseudoBoltzmann factor [19]

$$
W=\exp [-\beta P \Delta A+N \ln (1+\Delta A / A)],
$$

where $P$ is the pressure and $\Delta A$ is the difference in the area $\left(A \equiv L_{x} L_{y}\right.$ ) before and after the rescaling. In these simulations, the membrane is under tension, so we examine only negative pressures $P<0$.

For all but a few special cases, we set $N=144$ and generate 1000 configurations for a given point in parameter space. That is, 1000 configurations are generated for each $\beta P s_{\max }^{2}$ in the square-well case or for each $[P / k$, $\left.s_{0}(\beta k)^{-1 / 2}\right]$ combination in the Hooke's-law case. Each configuration is separated by a time $\tau \equiv N\left(l_{s} / l\right)^{2}$ Monte Carlo sweeps. Each initialization for a given parameter set is allowed to relax for $10 \tau$ before sample collection commences. More than $5 \times 10^{6}$ attempted moves are made on each vertex in a given set of configurations. The entire simulation required approximately $10 \mathrm{CPU}$ months on a 33-MHz MIPS $R 3000$ processor.

\section{APPENDIX B: ELASTIC CONSTANTS VIA FLUCTUATIONS}

The elastic constants of a two-dimensional material can be determined as follows. Two independent determinations of Young's modulus, $Y_{x}$ and $Y_{y}$, are made from fluctuations of the rectangle length in the $x$ and $y$ directions respectively:

$$
\begin{aligned}
& \beta Y_{x}=\left[\langle A\rangle\left(\left\langle L_{x}^{2}\right\rangle /\left\langle L_{x}\right\rangle^{2}-1\right)\right]^{-1}, \\
& \beta Y_{y}=\left[\langle A\rangle\left(\left\langle L_{y}^{2}\right\rangle /\left\langle L_{y}\right\rangle^{2}-1\right)\right]^{-1} .
\end{aligned}
$$

The area compression modulus $K_{A}$ is determined from the area fluctuations

$$
\beta K_{A}=\langle A\rangle /\left(\left\langle A^{2}\right\rangle-\langle A\rangle^{2}\right) .
$$

Finally, the shear modulus $\mu$ is obtained from $Y$ and $K_{A}$ via

$$
\mu=Y K_{A} /\left(4 K_{A}-Y\right),
$$

where the average of $Y_{x}$ and $Y_{y}$ is used for $Y$ in Eq. (B4). For the mean-field estimates quoted in Sec. III A, we replace $L_{x}$ by $s_{1}$ and $L_{y}$ by $s_{2} \sin \phi$. Mean values such as $\left\langle s_{1}^{2}\right\rangle$ can then be obtained easily by three-dimensional numerical integration of expressions such as Eq. (9). 
[1] R. Lipowsky, Nature 349, 475 (1991).

[2] J. Ambjørn, B. Durhuus, J. Fröhlich, and T. Jonsson, J. Stat. Phys. 55, 29 (1989); C. F. Baillie and D. A. Johnston, Phys. Lett. B 273, 380 (1991), and references therein.

[3] D. R. Nelson and L. Peliti, J. Phys. (Paris) 48, 1085 (1987).

[4] Y. Kantor and D. R. Nelson, Phys. Rev. A 36, 4020 (1987); see also Y. Kantor, ibid. 39, 6582 (1989).

[5] J. A. Aronovitz and T. C. Lubensky, Phys. Rev. Lett. 60, 2634 (1988).

[6] R. Lipowsky and M. Girardet, Phys. Rev. Lett. 65, 2893 (1990).

[7] D. H. Boal, U. Seifert, and A. Zilker, Phys. Rev. Lett. 69, 3405 (1992).

[8] S. Feng and P. N. Sen, Phys. Rev. Lett. 52, 216 (1984); S. Feng, M. F. Thorpe, and E. Garboczi, Phys. Rev. B 31, 276 (1985).

[9] Y. Kantor and I. Webman, Phys. Rev. Lett. 52, 1891 (1984).

[10] M. J. Saxton, Biophys. J. 57, 1167 (1990).
[11] D. R. Nelson and L. Radzihovsky, Europhys. Lett. 16, 79 (1991).

[12] D. H. Boal, Phys. Rev. E 47, 4604 (1993).

[13] D. Nelson (private communication).

[14] K. E. Evans, J. Phys. D 22, 1870 (1989); B. D. Caddock and K. E. Evans, ibid. 22, 1877 (1989); K. L. Alderson and K. E. Evans, Polymer 33, 4435 (1992).

[15] G. Wei and S. F. Edwards, Comput. Polymer Sci. 2, 44 (1992).

[16] K. E. Evans, M. A. Nkansah, I. J. Hutchinson, and S. C. Rogers, Nature 353, 124 (1991).

[17] R. S. Lakes, Science 235, 1038 (1987).

[18] Y. Kantor, M. Kardar, and D. R. Nelson, Phys. Rev. Lett. 57, 791 (1986)

[19] W. W. Wood, J. Chem. Phys. 48, 415 (1968); see also J. P. Hansen and I. R. McDonald, Theory of Simple Liquids (Oxford University Press, New York, 1986).

[20] See, for example, L. D. Landau and E. M. Lifshitz, Theory of Elasticity (Pergamon, London, 1959). 


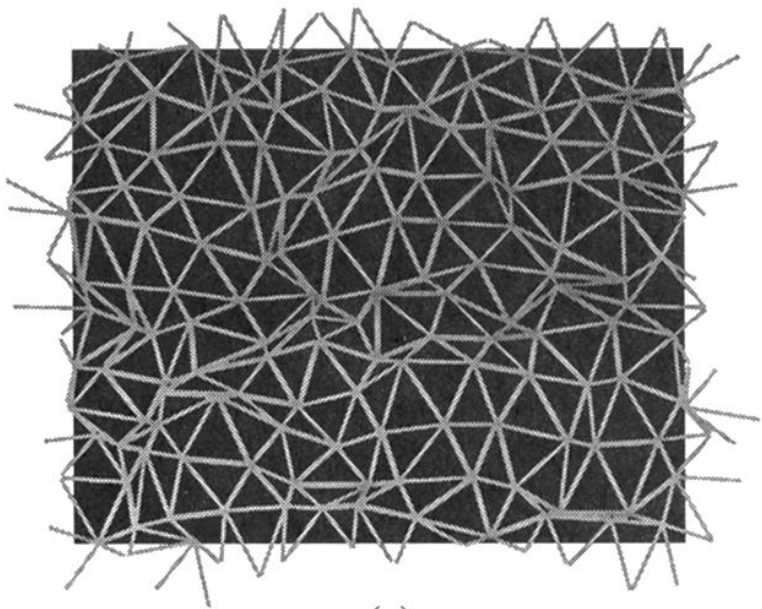

(a)

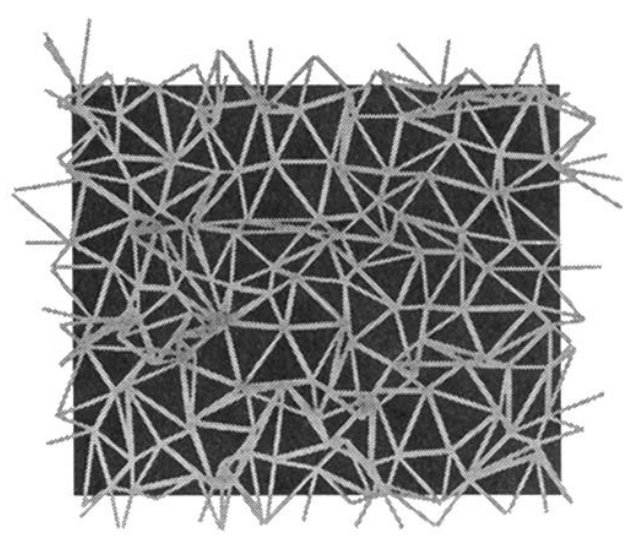

(b)

FIG. 1. Sample configurations for square-well potential interactions. Part (a) is a self-avoiding network while part (b) is not self-avoiding. Both networks were simulated at $\beta P s_{\max }^{2}=-4$, have $N=144$ vertices, and are drawn to the same scale. 


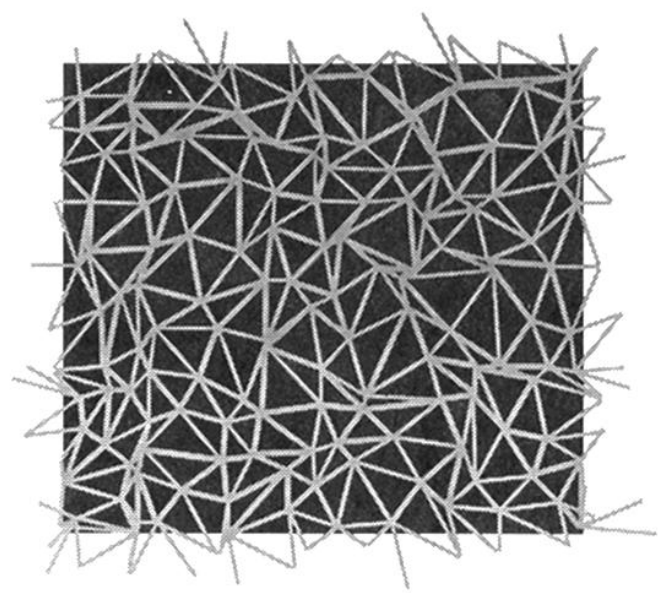

(a)

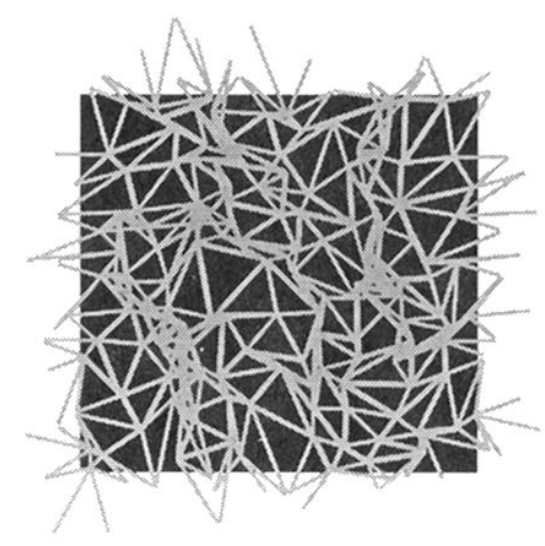

(b)

FIG. 14. Sample configurations for Hooke's-law networks. Part (a) is a self-avoiding network at $-P / k=0.8$ while part (b) is a phantom network at $-P / k=0.7$. Both networks have $N=144$ vertices and are drawn to the same scale. 